\title{
General Integral Control Design via Singular Perturbation Technique
}

\author{
Baishun Liu, Xiangqian Luo, Jianhui Li \\ Academy of Naval Submarine, Qingdao, China \\ Email: baishunliu@163.com, qdqtlxq@sina.com, jianhui_li@163.com
}

Received 10 August 2014; revised 8 September 2014; accepted 15 September 2014

Copyright (C) 2014 by authors and Scientific Research Publishing Inc.

This work is licensed under the Creative Commons Attribution International License (CC BY). http://creativecommons.org/licenses/by/4.0/

c) (i) Open Access

\section{Abstract}

This paper proposes a systematic method to design general integral control with the generic integrator and integral control action. No longer resorting to an ordinary control along with a known Lyapunov function, but synthesizing singular perturbation technique, mean value theorem, stability theorem of interval matrix and Lyapunov method, a universal theorem to ensure regionally as well as semi-globally asymptotic stability is established in terms of some bounded information. Its highlight point is that the error of integrator output can be used to stabilize the system, just like the system state, such that it does not need to take an extra and special effort to deal with the integral dynamic. Theoretical analysis and simulation results demonstrated that: general integral controller, which is tuned by this design method, has super strong robustness and can deal with nonlinearity and uncertainties of dynamics more forcefully.

\section{Keywords}

General Integral Control, Nonlinear Control, Robust Control, General Integrator, General Integral Action, Singular Perturbation Method, Output Regulation

\section{Introduction}

Integral control [1] plays an important role in practice because it ensures asymptotic tracking and disturbance rejection when exogenous signals are constants or planting parametric uncertainties appear. However, integral control design is not trivial matter because it depends on uncertain parameters and disturbances. Therefore, it is of important significance to develop the design method on the integral control.

In 2009, for overcoming the restriction of traditional integral control, the idea of general integral control firstly was proposed by [1], which presented some general integrators and controllers. However, their justification was not verified by mathematical analysis. General integral control designs based on linear system theory, 
sliding mode technique and feedback linearization technique were presented by [2]-[4], respectively. The main shortage of these design methods proposed by literature [2]-[4] is that they were all achieved by using a kind of particular integrator and linear integral action, which are a serious obstruction to design a high performance integral controller. In addition, general concave integral control [5], general convex integral control [6], constructive general bounded integral control [7] and the generalization of the integrator and integral control action [8] were all developed by resorting to an ordinary control along with a known Lyapunov function. This results in that design methods presented by [5]-[8] are all suspended in midair. Thus, it is a very valuable and challenging problem to establish a solid foundation for designing general integral control with the generic integrator and integral control action.

Motivated by the cognition above, this paper proposes a systematic method to design general integral control with the generic integrator and integral control action. The main contributions are that: 1) By mean value theorem, the nonlinear actions in the subsystem and integral dynamics are all reformulated as the linear forms on the interval matrix such that stability theorem of interval matrix can be used to deal with them; 2) The error of integrator output can be used to stabilize the system, just like the system state, such that it does not need to take an extra and special effort to deal with the integral dynamic; 3) No longer resorting to an ordinary control along with a known Lyapunov function, but synthesizing singular perturbation technique, mean value theorem, stability theorem of interval matrix and Lyapunov method, a universal theorem to ensure regionally as well as semiglobally asymptotic stability is established in terms of some bounded information. Consequently, this universal theorem is not suspended in midair but is developed with a solid foundation. Moreover, simulation results showed that general integral controller, which is tuned by this design method, has superstrong robustness and can deal with nonlinearity and uncertainties of dynamics more forcefully.

Throughout this paper, we use the notation $\lambda_{m}(A)$ and $\lambda_{M}(A)$ to indicate the smallest and largest eigenvalues, respectively, of a symmetric positive defined bounded matrix $A(x)$, for any $x \in R^{n}$. The norm of vector $x$ is defined as $\|x\|=\sqrt{x^{\mathrm{T}} x}$, and that of matrix $A$ is defined as the corresponding induced norm $\|A\|=\sqrt{\lambda_{M}\left(A^{\mathrm{T}} A\right)}$. For two $n \times m$ matrices $A$ and $B, A \geq B$ denotes element-by-element inequality. A family of interval matrices is defined as,

$$
\Lambda(\underline{A}, \bar{A})=\left[A \in R^{n \times m}: \underline{A} \leq A \leq \bar{A}\right]
$$

where $\underline{A}=\left[\underline{a}_{i j}\right]$ and $\bar{A}=\left[\bar{a}_{i j}\right]$ are fixed matrices. The family $\Lambda$ is described geometrically as hyperrectangle in the space $R^{n \times m}$ of the coefficients $a_{i j}$. We say that a $R^{n \times n}$ family matrix $\Lambda$ is Hurwitz stable if every $A \in \Lambda$ is Hurwitz stable.

The remainder of the paper is organized as follows: Section 2 describes the system under consideration, assumption and definition. Section 3 addresses the design method. Example and simulation are provided in Section 4. Conclusions are presented in Section 5.

\section{Problem Formulation}

Consider the following controllable nonlinear system,

$$
\left\{\begin{array}{l}
\dot{x}=f_{x}(x, z) \\
\dot{z}=f_{z}(x, z, w)+g(x, z, w) u
\end{array}\right.
$$

where $x \in R^{n}$ and $z \in R^{m}$ are the states; $u \in R^{m}$ is the control input; $w \in R^{l}$ is a vector of unknown constant parameters and disturbances. The partial derivative of function $f_{x}$ on $(x, z)$ is bounded in the control domain $D_{x} \times D_{z} \subset R^{n} \times R^{m}$, and $f_{x}(0,0)=0$. The functions, $f_{z}$ and $g$ are continuous in $(x, z, w)$ on the control domain $D_{x} \times D_{z} \times D_{w} \subset R^{n} \times R^{m} \times R^{l}$. We want to design a control law, $u$ such that $x(t) \rightarrow 0$ and $z(t) \rightarrow 0$ as $t \rightarrow \infty$.

Assumption 1: There is a unique pair $\left(0,0, u_{0}\right)$ that satisfies the equations,

$$
0=f_{z}(0,0, w)+g(0,0, w) u_{0}
$$

so that $x=z=0$ is the desired equilibrium point and $u_{0}$ is the steady-state control that is needed to maintain equilibrium at $x=z=0$. 
Assumption 2: No loss of generality, suppose that the functions $f_{z}(x, z, w)$ and $g(x, z, w)$ satisfies the following inequalities,

$$
\begin{gathered}
\left\|f_{z}(x, z, w)-f_{z}(0,0, w)\right\| \leq l_{f_{z}}^{x}\|x\|+l_{f_{z}}^{z}\|z\| \\
\bar{g} \geq g(x, z, w) \geq \underline{g}>0 \\
\|g(x, z, w)-g(0,0, w)\| \leq l_{g}^{x}\|x\|+l_{g}^{z}\|z\|
\end{gathered}
$$

for all $x \in D_{x}, \quad z \in D_{z}$ and $w \in D_{w}$. where $l_{f_{z}}^{x}, l_{f_{z}}^{z}, l_{g}^{x}$ and $l_{g}^{z}$ are all positive constants.

Definition 1: $F_{\phi}\left(a_{\phi}, b_{\phi}, c_{\phi}, x\right)$ with $a_{\phi}>0, b_{\phi}>0$ and $x \in R^{n}$ denotes the set of all continuously differential increasing functions [8],

$$
\phi(x)=\left[\begin{array}{llll}
\phi_{1}\left(x_{1}\right) & \phi_{2}\left(x_{2}\right) & \cdots & \phi_{n}\left(x_{n}\right)
\end{array}\right]^{\mathrm{T}}
$$

such that

$$
\begin{aligned}
& \phi(0)=0, \\
& \left|\phi_{i}\left(x_{i}\right)\right| \geq b_{\phi} \quad \forall x_{i} \in R:\left|x_{i}\right|>a_{\phi} \\
& c_{\phi} \geq \mathrm{d} \phi_{i}\left(x_{i}\right) / \mathrm{d} x_{i}>0 \quad \forall x_{i} \in R(i=1,2, \cdots, n) .
\end{aligned}
$$

where $|\cdot|$ stands for the absolute value.

Figure 1 depicts the example curves of one component of the functions belonging to the function set $F_{\phi}$. For instance, for all $x \in R$, the functions, $a x(a>0), \tanh (x), \operatorname{arcsinh}(x)$ and so on, all belong to function set $F_{\phi}$.

Definition 2: $F_{v}\left(c_{v}, x, z\right)$ with $c_{v}>0, x \in D_{x} \subset R^{n}$ and $z \in D_{z} \subset R^{m}$ denotes the set of all integrable functions [8],

$$
v(x, z)=\left[\begin{array}{llll}
v_{1}(x, z) & v_{2}(x, z) & \cdots & v_{n}(x, z)
\end{array}\right]^{\mathrm{T}}
$$

such that

$$
\begin{aligned}
& v_{i}(x, z)=\left.\frac{\partial v_{i}(x, z)}{\partial x}\right|_{(x, z)=\left(s_{i}^{x}, s_{i}^{Z}\right)} x+\left.\frac{\partial v_{i}(x, z)}{\partial z}\right|_{(x, z)=\left(s_{i}^{x}, s_{i}^{Z}\right)} z, \\
& c_{v} \geq\left.\frac{\partial v_{i}(x)}{\partial x}\right|_{(x, z)=\left(s_{i}^{x}, s_{i}^{z}\right)},\left.\frac{\partial v_{i}(x)}{\partial z}\right|_{(x, z)=\left(s_{i}^{x}, s_{i}^{z}\right)}>0
\end{aligned}
$$

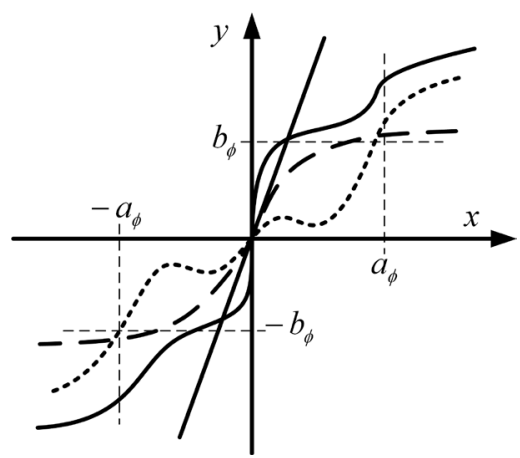

Figure 1. Example curves of one component of the functions belonging to the function set $F_{\phi}$. 
hold for all $i=1,2, \cdots, n$, and $\left(\varsigma_{i}^{x}, \varsigma_{i}^{z}\right)$ is a point on the line segment connecting $(x, z)$ to the origin.

Figure 2 depicts the example curves of one component of the functions belonging to the function set $F_{v}$. For instance, for all $x \in(-1,1)$, and $z \in(-1,1)$, the functions, $x+3 z, \tanh (x)+\sinh (z), \sinh (x+2 z)$, and so on, all belong to the function set $F_{v}$.

\section{Control Design}

In general, integral controller comprises three components: the stabilizing controller, the integral control action and the integrator, and then the general integral controller can be given as,

$$
\left\{\begin{array}{l}
u=-\varepsilon^{-1}\left(K_{x} x+K_{z} z+K_{\sigma} \phi(\sigma)\right) \\
\dot{\sigma}=\beta(\sigma) v(x, z)
\end{array}\right.
$$

where $K_{x}, K_{z}$ and $K_{\sigma}$ are the $m \times n, m \times m$ and $m \times m$ gain matrices, respectively; $\dot{\sigma}_{i}=\beta_{i}\left(\sigma_{i}\right) v_{i}(x, z)$; $c_{\beta} \geq \beta_{i}\left(\sigma_{i}\right)>0 \quad(i=1,2, \cdots, m) ; \varepsilon$ is a positive constant; the functions $\phi(\bullet)$ and $v(\bullet)$ belong to the function sets $F_{\phi}$ and $F_{v}$, respectively.

Thus, substituting (6) into (1), obtain the augmented system,

$$
\left\{\begin{array}{l}
\dot{x}=f_{x}(x, z) \\
\varepsilon \dot{z}=\varepsilon f_{z}(x, z, w)-g(x, z, w)\left(K_{x} x+K_{z} z+K_{\sigma} \phi(\sigma)\right) \\
\dot{\sigma}=\beta(\sigma) v(x, z)
\end{array}\right.
$$

By Assumption 1 and choosing $\varepsilon^{-1} K_{\sigma}$ to be nonsingular and large enough, and then setting $\dot{Z}=0$ and $x=z=0$ of the system (7), we obtain,

$$
g(0,0, w) K_{\sigma} \phi\left(\sigma_{0}\right)=\varepsilon f_{z}(0,0, w)
$$

Therefore, we ensure that there is a unique solution $\sigma_{0}$, and then $\left(0,0, \sigma_{0}\right)$ is a unique equilibrium point of the closed-loop system (7) in the domain of interest. At the equilibrium point, $x=z=0$, irrespective of the value of $w$.

Now, by Mean Value Theorem for each component of the vector function $f_{x}(x, z)$, we have,

$$
f_{x i}(x, z)=\left.\frac{\partial f_{x i}(x, z)}{\partial x}\right|_{(x, z)=\left(s_{i}^{x}, \varsigma_{i}^{z}\right)} x+\left.\frac{\partial f_{x i}(x, z)}{\partial z}\right|_{(x, z)=\left(\varsigma_{i}^{x}, \varsigma_{i}^{z}\right)} z
$$

where $i=1,2, \cdots, n$ and $\left(\varsigma_{i}^{x}, \varsigma_{i}^{z}\right)$ is a point on the line segment connecting $(x, z)$ to the origin.

For convenience, the function, $f_{x}(x, z)$ can be written as a compact formulation, that is,

$$
f_{x}(x, z)=\theta_{f_{x}}^{x} x+\theta_{f_{x}}^{z} z
$$

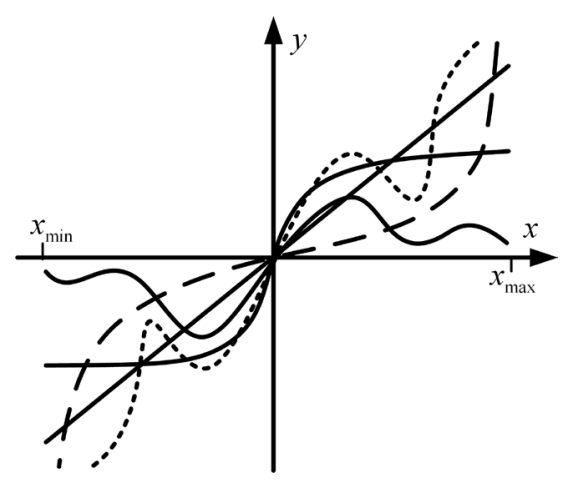

Figure 2. Example curves of one component of the functions belonging to the function set $F_{v}$. 
where,

$$
\theta_{f_{x}}^{x}=\left[\begin{array}{llll}
\theta_{f_{x 1}}^{x} & \theta_{f_{x 2}}^{x} & \cdots & \theta_{f_{x n}}^{x}
\end{array}\right]^{\mathrm{T}}, \quad \theta_{f_{x}}^{z}=\left[\begin{array}{llll}
\theta_{f_{x 1}}^{z} & \theta_{f_{x 2}}^{2} & \cdots & \theta_{f_{x n}}^{z}
\end{array}\right]^{\mathrm{T}} .
$$

Thus, by the bound of partial derivative of function $f_{x}$ on $(x, z)$, we can ensure that the matrices $\theta_{f_{x}}^{x}$ and $\theta_{f_{x}}^{z}$ belong to the families of interval matrices, respectively, that is,

$$
\theta_{f_{x}}^{x} \in\left[\underline{\theta}_{f_{x}}^{x}, \bar{\theta}_{f_{x}}^{x}\right], \quad \theta_{f_{x}}^{z} \in\left[\underline{\theta}_{f_{x}}^{z}, \bar{\theta}_{f_{x}}^{z}\right] .
$$

In the same way, we obtain,

$$
\beta(\sigma) v(x, z)=\theta_{v}^{x} x+\theta_{v}^{z} z, \quad \phi(\sigma)-\phi\left(\sigma_{0}\right)=\theta_{\varphi}\left(\sigma-\sigma_{0}\right)
$$

Therefore, by $c_{\beta} \geq \beta_{i}\left(\sigma_{i}\right)>0$, and Definitions 1 and 2, we have,

$$
\theta_{v}^{x} \in\left[\underline{\theta}_{v}^{x}, \bar{\theta}_{v}^{x}\right], \quad \theta_{v}^{z} \in\left[\underline{\theta}_{v}^{z}, \bar{\theta}_{v}^{z}\right], \quad \theta_{\phi} \in\left[\underline{\theta}_{\phi}, \bar{\theta}_{\phi}\right],
$$

and then substituting them and (8) into (7), obtain,

$$
\left\{\begin{array}{l}
\dot{x}=\theta_{f_{x}}^{x} x+\theta_{f_{x}}^{z} z \\
\varepsilon \dot{z}=-g K_{x} x-g K_{z} z-g K_{\sigma} \theta_{\phi}\left(\sigma-\sigma_{0}\right)+\varepsilon\left(f_{z}-f_{z 0}\right)-\varepsilon\left(g-g_{0}\right) g_{0}^{-1} f_{z 0} \\
\dot{\sigma}=\theta_{v}^{x} x+\theta_{v}^{z} z
\end{array}\right.
$$

where

$$
f_{z}=f_{z}(x, z, w), f_{z 0}=f_{z}(0,0, w), g=g(x, z, w) \text {, and } g_{0}=g(0,0, w) \text {. }
$$

Now, defining $\eta=\left[\begin{array}{ll}x & \sigma-\sigma_{0}\end{array}\right]^{T}, \quad y=z-h(\eta)$,

$$
h(\eta)=-K_{z}^{-1} K_{x} x-K_{z}^{-1} K_{\sigma} \theta_{\phi}\left(\sigma-\sigma_{0}\right),
$$

and then the closed-loop system (9) can be rewritten as,

$$
\left\{\begin{array}{l}
\dot{\eta}=A \eta+\delta_{\eta}(y) \\
\frac{\mathrm{d} y}{\mathrm{~d} \tau}=\Lambda y+\varepsilon \delta_{y}(\eta, y)
\end{array}\right.
$$

where

$$
\begin{gathered}
A=\left[\begin{array}{cc}
\theta_{f_{x}}^{x}-\theta_{f_{x}}^{z} K_{z}^{-1} K_{x} & -\theta_{f_{x}}^{z} K_{z}^{-1} K_{\sigma} \theta_{\phi} \\
\theta_{v}^{x}-\theta_{v}^{z} K_{z}^{-1} K_{x} & -\theta_{v}^{z} K_{z}^{-1} K_{\sigma} \theta_{\phi}
\end{array}\right], \\
\delta_{\eta}(y)=\left[\begin{array}{cc}
\theta_{f_{x}}^{z} & 0 \\
\theta_{v}^{z} & 0
\end{array}\right]\left[\begin{array}{l}
y \\
0
\end{array}\right],
\end{gathered}
$$

$\Lambda=-g K_{z}, \quad \tau=t / \varepsilon$, and

$$
\delta_{y}(\eta, y)=\left(f_{z}-f_{z 0}\right)-\left(g-g_{0}\right) g_{0}^{-1} f_{z 0}-\dot{h}(\eta) .
$$

In the absence of $\delta_{\eta}(y)$ and $\delta_{y}(\eta, y)$, the asymptotic stability of the closed-loop system (10) can be achieved by designing the interval matrices $A$ and $\Lambda$ are all Hurwitz stable [9]. Thus, by linear system theory, two quadratic Lyapunov functions,

$$
\begin{aligned}
& V_{\eta}(\eta)=\eta^{\mathrm{T}} P_{A} \eta \\
& V_{y}(y)=y^{\mathrm{T}} P_{\Lambda} y
\end{aligned}
$$


can be obtained, respectively. Where $P_{A}$ and $P_{\Lambda}$ are the solutions of Lyapunov equations $P_{A} A+A^{\mathrm{T}} P_{A}=-I$ and $P_{\Lambda} \Lambda+\Lambda^{\mathrm{T}} P_{\Lambda}=-I$, respectively. It is obvious that $P_{A}$ and $P_{\Lambda}$ are all interval matrices, that is,

$$
P_{A} \in\left[\underline{P}_{A}, \bar{P}_{A}\right] \text { and } P_{\Lambda} \in\left[\underline{P}_{\Lambda}, \bar{P}_{\Lambda}\right] \text {. }
$$

Based on the Lyapunov Functions (11) and (12), a composite Lyapunov function candidate [10] for the closed-loop system (10) can be written as,

$$
V(\eta, y)=(1-d) V_{\eta}(\eta)+d V_{y}(y) \quad 0<d<1
$$

Obviously, Lyapunov function candidate (13) is positive define. Therefore, our task is to show that its time derivative along the trajectories of the closed-loop system (10) is negative define, which is given by,

$$
\begin{aligned}
\dot{V}(\eta, y) & =(1-d) \dot{V}_{\eta}(\eta)+d \dot{V}_{y}(y) \\
& =-(1-d) \eta^{\mathrm{T}} \eta+(1-d) \eta^{\mathrm{T}} P_{A} \delta_{\eta}(y)+(1-d) \delta_{\eta}^{\mathrm{T}}(y) P_{A} \eta-d y^{\mathrm{T}} y+d y^{\mathrm{T}} P_{\Lambda} \delta_{y}(\eta, y)+d \delta_{y}^{\mathrm{T}}(\eta, y) P_{A} y .
\end{aligned}
$$

By definitions of $h(\eta)$ and $\phi(\sigma)$, we have,

$$
\begin{gathered}
\dot{h}(\eta)=-K_{z}^{-1} K_{x} \dot{x}-K_{z}^{-1} K_{\sigma} \dot{\phi}(\sigma) \\
\dot{\phi}(\sigma)=\frac{\partial \phi(\sigma)}{\partial \sigma} \dot{\sigma}=\frac{\partial \phi(\sigma)}{\partial \sigma} \beta(\sigma) v(x, z)
\end{gathered}
$$

and then substituting $\dot{\phi}(\sigma), \dot{x}$, and $z=y+h(\eta)$ into $\dot{h}(\eta)$, and using Assumptions 2, and Definitions 1 and 2, we have,

$$
\left\|\delta_{y}(\eta, y)\right\| \leq \gamma_{\delta_{y}}^{\eta}\|\eta\|+\gamma_{\delta_{y}}^{y}\|y\|
$$

In addition, by $\theta_{f_{x}}^{z} \in\left[\underline{\theta}_{f_{x}}^{z}, \bar{\theta}_{f_{x}}^{z}\right], \theta_{v}^{z} \in\left[\underline{\theta}_{v}^{z}, \bar{\theta}_{v}^{z}\right]$ and definition of $\delta_{\eta}(y)$, obtain,

$$
\left\|\delta_{\eta}(y)\right\| \leq \gamma_{\delta_{\eta}}^{y}\|y\|
$$

where $\gamma_{\delta_{y}}^{\eta}, \gamma_{\delta_{y}}^{y}$, and $\gamma_{\delta_{\eta}}^{y}$ are all positive constants.

Substituting (15) and (16) into (14), obtain,

$$
\dot{V}(\eta, y) \leq-(1-d)\|\eta\|^{2}-d\left(\varepsilon^{-1}-\gamma\right)\|y\|^{2}+2\left((1-d) \beta_{1}+d \beta_{2}\right)\|y\|\|\eta\|=-\xi^{\mathrm{T}} Q \xi
$$

where

$$
\begin{aligned}
& \beta_{1}=\gamma_{\delta_{\eta}}^{y}\left\|P_{\eta}\right\|, \quad\left\|P_{\eta}\right\|=\operatorname{Max}_{P_{A} \in\left[\underline{P}_{A}, \bar{P}_{A}\right]}\left(\left\|P_{A}\right\|\right), \\
& \beta_{2}=\gamma_{\delta_{y}}^{\eta}\left\|P_{y}\right\|, \quad\left\|P_{y}\right\|=\operatorname{Max}_{P_{\Lambda} \in\left[\underline{P}_{A}, \bar{P}_{A}\right]}\left(\left\|P_{\Lambda}\right\|\right), \\
& \gamma=2 \gamma_{\delta_{y}}^{y}\left\|P_{y}\right\|, \quad \xi=[\|\eta\|\|y\|]^{\mathrm{T}}, \\
& Q=\left[\begin{array}{cc}
(1-d) & (d-1) \beta_{1}-d \beta_{2} \\
(d-1) \beta_{1}-d \beta_{2} & d\left(\varepsilon^{-1}-\gamma\right)
\end{array}\right] .
\end{aligned}
$$

The right-hand side of the inequality (17) is a quadratic form, which is negative define when,

$$
(1-d) d\left(\varepsilon^{-1}-\gamma\right)>\left((1-d) \beta_{1}+d \beta_{2}\right)^{2}
$$

which is equivalent to,

$$
\varepsilon<\varepsilon_{d}=\frac{(1-d) d}{(1-d) d \gamma+\left((1-d) \beta_{1}+d \beta_{2}\right)^{2}}
$$


By the dependence of $\varepsilon_{d}$ on $d$, it is obvious that the maximum value of $\varepsilon_{d}$ occurs at $d^{*}=\beta_{1} /\left(\beta_{1}+\beta_{2}\right)$ [10] and is given by,

$$
\varepsilon^{*}<\varepsilon_{d}=\frac{1}{\gamma+4 \beta_{1} \beta_{2}}
$$

It follows that the origin of closed-loop system (10) is asymptotically stable for all $\varepsilon<\varepsilon^{*}$. Consequently, by $\eta=0$ and $y=0$, we have $x=0, \sigma=\sigma_{0}$ and $z=h(\eta)=0$. This means that the closed-loop system (7) is asymptotically stable, too. This established the following Theorem.

Theorem 1: Under Assumptions 1 and 2, if there exist gain matrices $K_{x}, K_{z}$ and $K_{\sigma}$ such that the interval matrices $A$ and $\Lambda$ are all Hurwitz stable and the following inequality,

$$
\lambda_{m}\left(g_{m} K_{\sigma} \phi\left(a_{\phi}\right)\right)>\varepsilon\|f(0,0, w)\|
$$

holds to ensure that there exist positive constants $\varepsilon_{d}$ and $\varepsilon^{*}$, and then $\left(0,0, \sigma_{0}\right)$ is an exponentially stable equilibrium point of the closed-loop system (7) for all $\varepsilon<\varepsilon^{*}$. Moreover, if all assumptions hold globally, and then it is globally exponentially stable.

Discussion 1: It is not hard to see that: Just using singular perturbation technique, two key points in stability analysis are solved, that is, one is that it decomposes the whole system into two interconnection subsystems such that it is very easy to obtain two quadratic Lyapunov functions; another is that it derives the condition on the controller gains to ensure the asymptotic stability. Therefore, although mean value theorem, stability theorem of interval matrix, singular perturbation technique and Lyapunov method are all indispensable components, singular perturbation technique plays a decisive role. This is why our design method is called as singular perturbation one.

Discussion 2: By special concerns of the equation $\dot{\eta}$ and its matrix $A$, it is not hard to find that the error of integrator output $\sigma-\sigma_{0}$ appears in not only the system dynamic $\dot{x}$ but also the integral dynamic $\dot{\sigma}$. This results in that integral dynamic $\dot{\sigma}$ has the same formulation as the system dynamic $\dot{x}$. Thus, the error of integrator output can be used to stabilize the system, just like the system state $x$. This means that in stability analysis, the integral dynamic plays a positive role and it does not need to take an extra and special effort to deal with it. As a result, this is a highlight point of this paper.

Discussion 3: Compared with general integral control proposed by [2]-[8], the main differences are that: 1) The integrator and integral action here are all generalized by two function sets, respectively. However, they are all particular in [2]-[4]; 2) As like reference [7], the integrator here increases a positive define vector function $\beta(\sigma)$ on base of the integrator presented by [8], which can be used as an additional freedom of degree to improve the integrator performance. However, it is not completely freedom and mainly used to construct the bound condition in [7]; 3) Control design here is achieved by synthesizing singular perturbation technique, mean value theorem, stability theorem of interval matrix and Lyapunov method. However, in reference [2]-[8], the designs are achieved by linear system theory, sliding mode technique, feedback linearization technique and Lyapunov method, respectively; 4) For the stability analysis, the integral dynamic here not only plays a positive role but also its negative effects can be effectively attenuated by decreasing $\varepsilon$. However, the integral dynamics not only almost have not positive actions in [5]-[8] but also there no effective method was proposed to deal with its negative effects; 5) Theorem 1 is not suspended in midair but is established on a solid foundation. However, stability theorems [5]-[8] were all developed by resorting to an ordinary control along with a known Lyapunov function.

Discussion 4: From the design procedure above, it is obvious that: First, the equation $\dot{z}$ in (1) is transformed into the singular perturbation form such that singular perturbation technique can be used to attenuate the nonlinearities and/or uncertainties, $\delta_{y}(\eta, y)$. Second, by mean value theorem, the nonlinear terms, $f_{x}(x, z), \phi(\sigma)$ and $\beta(\sigma) v(x, z)$ are all reformulated as the linear forms on the interval matrix such that stability theorem of interval matrix can be used to deal with them. Finally, by Lyapunov method, a universal theorem to ensure regionally as well as semi-globally asymptotic stability is established in terms of some bounded information. All of them synthesize a systematic method to design general integral control with the generic integrator and integral control action. Specially, the error of integrator output can be used to stabilize the system, just like the system state $x$. All those mean that the design method here can more effectively deal with nonlinearity and uncertainty of dynamics, and then makes the engineers more easily design a stable controller. 


\section{Example and Simulation}

Consider the pendulum system [10] described by,

$$
\ddot{\theta}=-a \sin \theta-b \dot{\theta}+c T
$$

where $a, b, c>0, \theta$ is the angle subtended by the rod and the vertical axis, and $T$ is the torque applied to the pendulum. View $T$ as the control input and suppose we want to regulate $\theta$ to $r$. Now, taking $x=\theta-r$, $z=\dot{\theta}, k_{z}=3$ and $k_{x}=k_{\sigma}=9$, general integral controller can be written as,

$$
\left\{\begin{array}{l}
u=-\varepsilon^{-1}(9 x+3 z+9(\sigma+\tanh (\sigma))) \\
\dot{\sigma}=5 x+\sinh (x)+z+\tanh (z)
\end{array},\right.
$$

and then the pendulum system with the normal parameters $a=c=10$ and $b=1$ can be written as,

$$
\left\{\begin{array}{l}
\dot{\eta}=A \eta+\delta_{\eta}(y) \\
\frac{\mathrm{d} y}{\mathrm{~d} \tau}=-30 y+\varepsilon \delta_{y}(\eta, y)
\end{array}\right.
$$

where $A \in[\underline{A}, \bar{A}]$,

$$
\begin{gathered}
\underline{A}=\left[\begin{array}{cc}
-3 & -6 \\
0 & -12
\end{array}\right], \bar{A}=\left[\begin{array}{cc}
-3 & -3 \\
13.6 & -6
\end{array}\right], \\
\delta_{\eta}(y)=\left[\begin{array}{ll}
1 & 0 \\
2 & 0
\end{array}\right]\left[\begin{array}{l}
y \\
0
\end{array}\right], \\
\delta_{y}(\eta, y)=3 \dot{x}_{1}+3 \dot{\phi}(\sigma)-z-10(\sin (x+r)-\sin (r)) .
\end{gathered}
$$

By stability theorem of interval matrix [9], it is easy to verify that the interval matrix $A$ is Hurwitz stable. Thus, by solving Lyapunov equation $P_{A} A+A^{\mathrm{T}} P_{A}=-I$ and $P_{\Lambda} \Lambda+\Lambda^{\mathrm{T}} P_{\Lambda}=-I$, the maximum values of $\left\|P_{A}\right\|$ and $\left\|P_{\Lambda}\right\|$ can be obtained, respectively, that is,

$$
\left\|P_{\eta}\right\| \leq 0.177 \text { and }\left\|P_{y}\right\| \leq 0.034 \text {. }
$$

and then using $\left\|\delta_{y}(\eta, y)\right\| \leq 104.5\|\eta\|+14\|y\|$ and $\left\|\delta_{\eta}(y)\right\| \leq \sqrt{5}\|y\|$, we have,

$$
\beta_{1}=0.4, \beta_{2}=1.74 \text { and } \gamma=0.47 \text {. }
$$

Therefore, the stability of the closed-loop system (22) can be ensured for all $\varepsilon<0.3$ and $r \in[-\pi, \pi]$.

For illustrating the performance of controller above, the simulations are achieved under normal and perturbed parameters, respectively. The normal parameters are $a=c=10$ and $b=1$. In the perturbed case, $b$ and $c$ are reduced to 0.25 and 2.5 , respectively, corresponding to four times the mass.

Figure 3 showed the simulation results under normal (solid line) and perturbed (dashed line) cases. The following observations can be made: the optimum response in the whole domain of interest can all be achieved by a set of the same control gains, even under the case that four times payload changes. This demonstrates that although the design method here is too conservative, general integral controller, which is tuned by only the normal parameters, has superstrong robustness, fast convergence, and good flexibility and can deal with nonlinearity and uncertainties of dynamics more forcefully.

\section{Conclusions}

This paper proposes a systematic method to design general integral control with the generic integrator and integral control action. The main contributions are that: 1) By mean value theorem, the nonlinear actions in the subsystem and integral dynamics are all reformulated as the linear forms on the interval matrix such that stability theorem of interval matrix can be used to deal with them; 2) The error of integrator output can be used to 


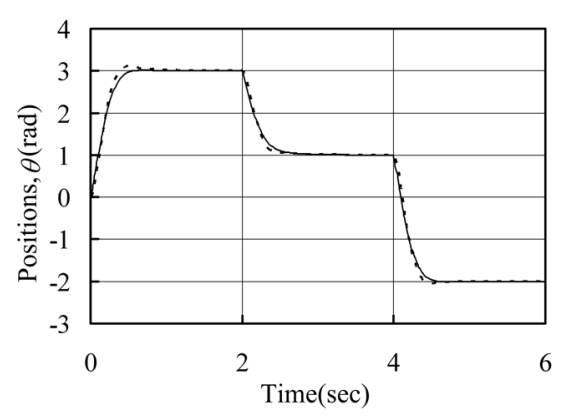

Figure 3. System output under normal (solid line) and perturbed case (dashed line).

stabilize the system, just like the system state, such that it does not need to take an extra and special effort to deal with the integral dynamic; 3) No longer resorting to an ordinary control along with a known Lyapunov function, but synthesizing singular perturbation technique, mean value theorem, stability theorem of interval matrix and Lyapunov method, a universal theorem to ensure regionally as well as semi-globally asymptotic stability is established in terms of some bounded information. Consequently, this universal theorem is not suspended in midair but is developed with a solid foundation.

Simulation results showed that general integral controller, which is tuned by this design method, has superstrong robustness and can deal with nonlinearity and uncertainties of dynamics more forcefully.

\section{References}

[1] Liu, B.S. and Tian, B.L. (2009) General Integral Control. Proceedings of the International Conference on Advanced Computer Control, Singapore, 22-24 January 2009, 136-143.

[2] Liu, B.S. and Tian, B.L. (2012) General Integral Control Design Based on Linear System Theory. Proceedings of the 3rd International Conference on Mechanic Automation and Control Engineering, Baotou, 27-29 July 2012, Vol. 5, 3174-3177.

[3] Liu, B.S. and Tian, B.L. (2012) General Integral Control Design Based on Sliding Mode Technique. Proceedings of the 3rd International Conference on Mechanic Automation and Control Engineering, Baotou, 27-29 July 2012, Vol. 5, 3178-3181.

[4] Liu, B.S., Li, J.H. and Luo, X.Q. (2014) General Integral Control Design via Feedback Linearization. Intelligent Control and Automation, 5, 19-23. http://dx.doi.org/10.4236/ica.2014.51003

[5] Liu, B.S., Luo, X.Q. and Li, J.H. (2013) General Concave Integral Control. Intelligent Control and Automation, 4, 356361. http://dx.doi.org/10.4236/ica.2013.44042

[6] Liu, B.S., Luo, X.Q. and Li, J.H. General Convex Integral Control. International Journal of Automation and Computing.

[7] Liu, B.S. (2014) Constructive General Bounded Integral Control. Intelligent Control and Automation, 5, 146-155. http://dx.doi.org/10.4236/ica.2014.53017

[8] Liu, B.S. (2014) On the Generalization of Integrator and Integral Control Action. International Journal of Modern Nonlinear Theory and Application, 3, 4452. http://dx.doi.org/10.4236/ijmnta.2014.32007

[9] Krans, F.J. and Mansour, M. (1991) Sufficient Conditions for Hurwitz and Schar Stability of Interval Matrices. Proceeding of the 30th conference on decision and control, Brighton, December 1991, 3043-3044.

[10] Khalil, H.K. (2007) Nonlinear Systems. 3rd Edition, Electronics Industry Publishing, Beijing, 449-453. 
Scientific Research Publishing (SCIRP) is one of the largest Open Access journal publishers. It is currently publishing more than 200 open access, online, peer-reviewed journals covering a wide range of academic disciplines. SCIRP serves the worldwide academic communities and contributes to the progress and application of science with its publication.

Other selected journals from SCIRP are listed as below. Submit your manuscript to us via either submit@scirp.org or Online Submission Portal.
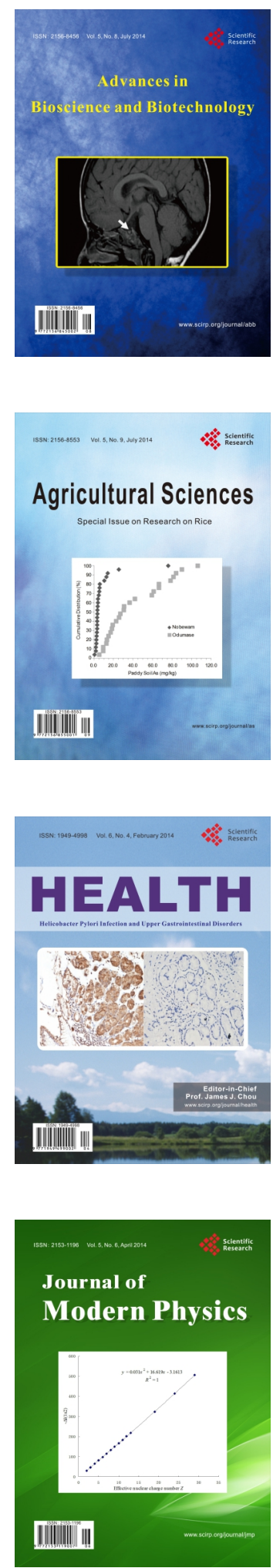
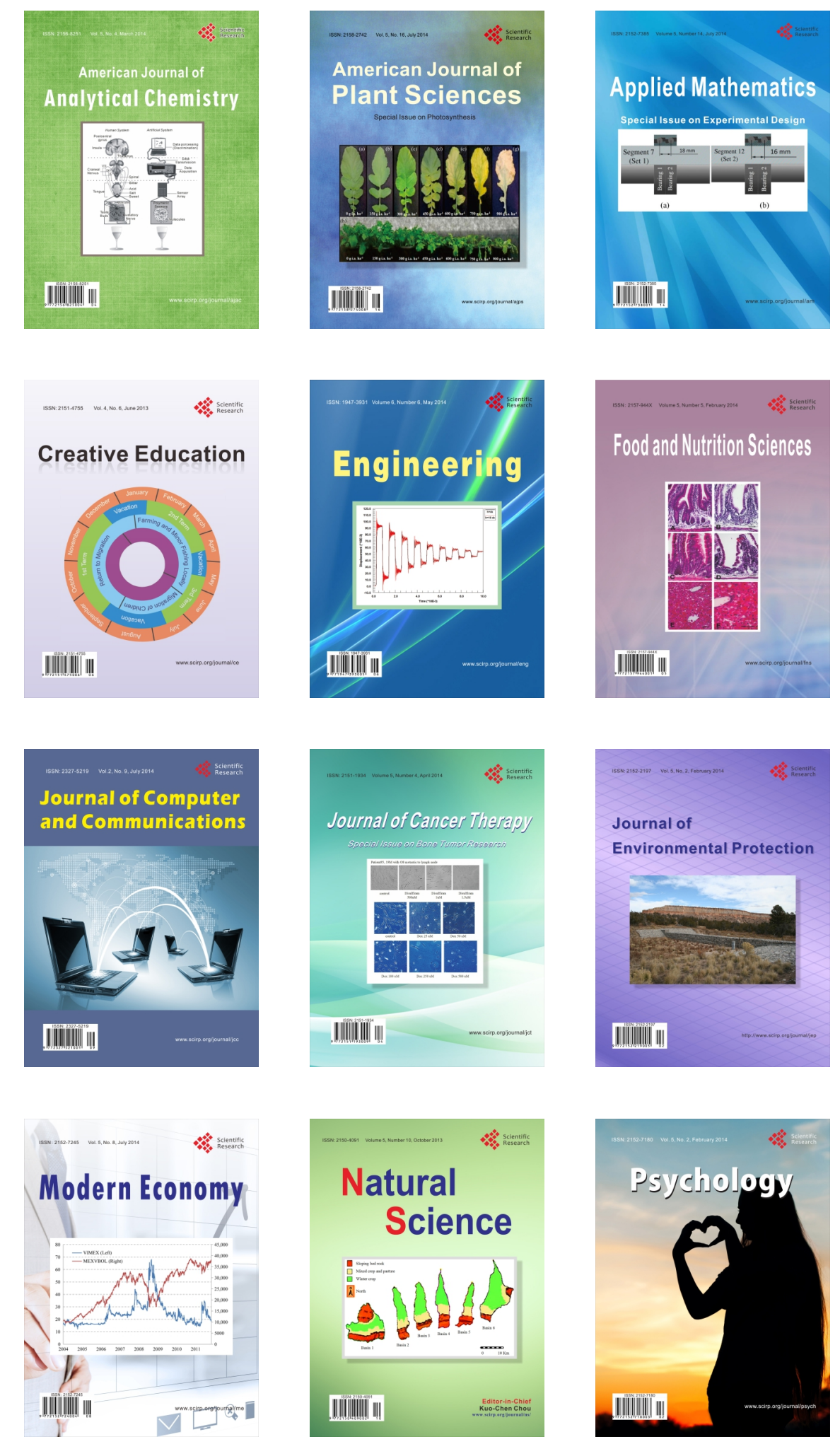